\section{An Unusual Association of Chronic Recurrent Multifocal Osteomyelitis, Pyoderma Gangrenosum, and Takayasu Arteritis}

To the Editor:

Chronic recurrent multifocal osteomyelitis (CRMO) is hypothesized to be an autoimmune disorder because of its association with multiple autoimmune diseases, including inflammatory bowel disease, psoriasis, acne, pustulosis, Sweet syndrome, dyserythropoietic anemia, pyoderma gangrenosum (PG), sclerosing cholangitis, inflammatory arthritis, Still disease, Takayasu arteritis (TA), Ollier disease, and dermatomyositis ${ }^{1,2}$.

PG association with TA is rare; however, Ujiie, et al showed that PG is associated with TA in $33 \%$ of patients ${ }^{3}$.

Occurrence of all 3 conditions together (CRMO, PG, and TA) is very rarely reported.

A 10-year-old girl born to nonconsanguineous parents presented with a history of intermittent swelling over the right side of the mandible, associated with pain and claudication pain over legs and back with breathlessness on exertion for the past 2 years. She also developed ulcerative cauliflower-like skin lesions over the dorsum of left foot over the past 2 months (Figure 1). On examination, her pulses were absent in the carotid, brachial, radial, popliteal, and dorsalis pedis on left side, and the right femoral, popliteal, and dorsalis pedis. Right-sided carotid bruit was heard. Cauliflower-like skin lesion was present over the dorsum of left foot. She had a bony swelling of the body of the right mandible. The rest of the systemic examination was unremarkable. Ethics approval was not required in accordance with the policy of our institution and the patient's father's written informed consent to publish was obtained.

Her investigations revealed hemoglobin of $9.1 \mathrm{gm} / \mathrm{dl}$ with total count of 13,900 cells $/ \mathrm{mm}^{3}$ and platelets of 503,000 cells $/ \mathrm{mm}^{3}$. Her erythrocyte sedimentation rate (ESR) was $57 \mathrm{~mm} / \mathrm{h}$ and C-reactive protein (CRP) was $122 \mathrm{mg} / \mathrm{l}$. Her renal and liver function tests were normal. Serum complements were normal and antinuclear antibody was negative. Routine, fungal, and acid-fast bacillus culture from the ulcerative lesion were sterile. Bone scan showed increased pooling of tracer in the mandible region, suggestive of subacute inflammation. Bone biopsy from the mandible was consistent with chronic osteomyelitis. Skin biopsy was suggestive of PG. Her
QuantiFERON Gold (Interferon Gamma assay), Mantoux, and mycobacterium culture from biopsy were negative. Radiograph of the right tibia demonstrated a lytic lesion in the metaphysis of the right distal tibia. However, she was asymptomatic.

Magnetic resonance angiogram showed dilation of ascending aorta and narrowed left common carotid artery. Left subclavian, right subclavian, and axillary were narrowed at origin and proximal portion. Multiple collaterals were seen in the region of the axilla with non-visualization of the axillary artery. About $50 \%$ of mild wall thickening was seen in the proximal right common carotid artery. There was narrowing at lower thoracic aorta with mild dilation just above the level of the diaphragm. Abdominal aorta showed wall thickening with a beaded appearance with multiple areas of narrowing and intervening areas appearing dilated with sacculated appearance. Celiac artery appears mildly narrowed. Renal arteries revealed high-grade stenosis at the origin of the left renal artery with mild post-stenotic dilation. With all features, she was diagnosed with Type V TA (Figure 1).

With all clinical, radiological, and pathological findings, she was diagnosed with CRMO, PG, and TA. She started treatment with prednisolone $(1 \mathrm{mg} / \mathrm{kg} /$ day) and mycophenolate mofetil. After 3 months of starting immunosuppression, she underwent coronary angiogram with ballooning of bilateral common femoral artery and stenting of left subclavian artery. At followup after 6 months, her skin lesions had healed and mandibular swelling (Figure 2) had also come down, along with normalization of laboratory variables such as CRP and ESR.

Interestingly, in our patient, $\mathrm{CRMO}, \mathrm{PG}$, and $\mathrm{TA}-$ all 3 pathologies occurred in a sequential manner, initially symptoms of CRMO followed by TA and subsequently PG. Occurrence of the 3 conditions together is rare: In the literature to date, 2 similar cases have been reported. In the study by Dagan, et al with a similar pattern of occurrence ${ }^{4}$ and in a study by Ghosn, et $a l^{5}$, a similar clinical spectrum of TA, malignant PG, and relapsing polychondritis was reported. Both these patients' treatments with steroids and immunomodulators resulted in dramatic response.

The exact reason or genetic basis for association of all these inflammatory condition is still obscure. Hence, followup of all patients with CRMO, TA, and PG for association with one another for early diagnosis and prompt treatment with immunomodulators will be of immense importance.

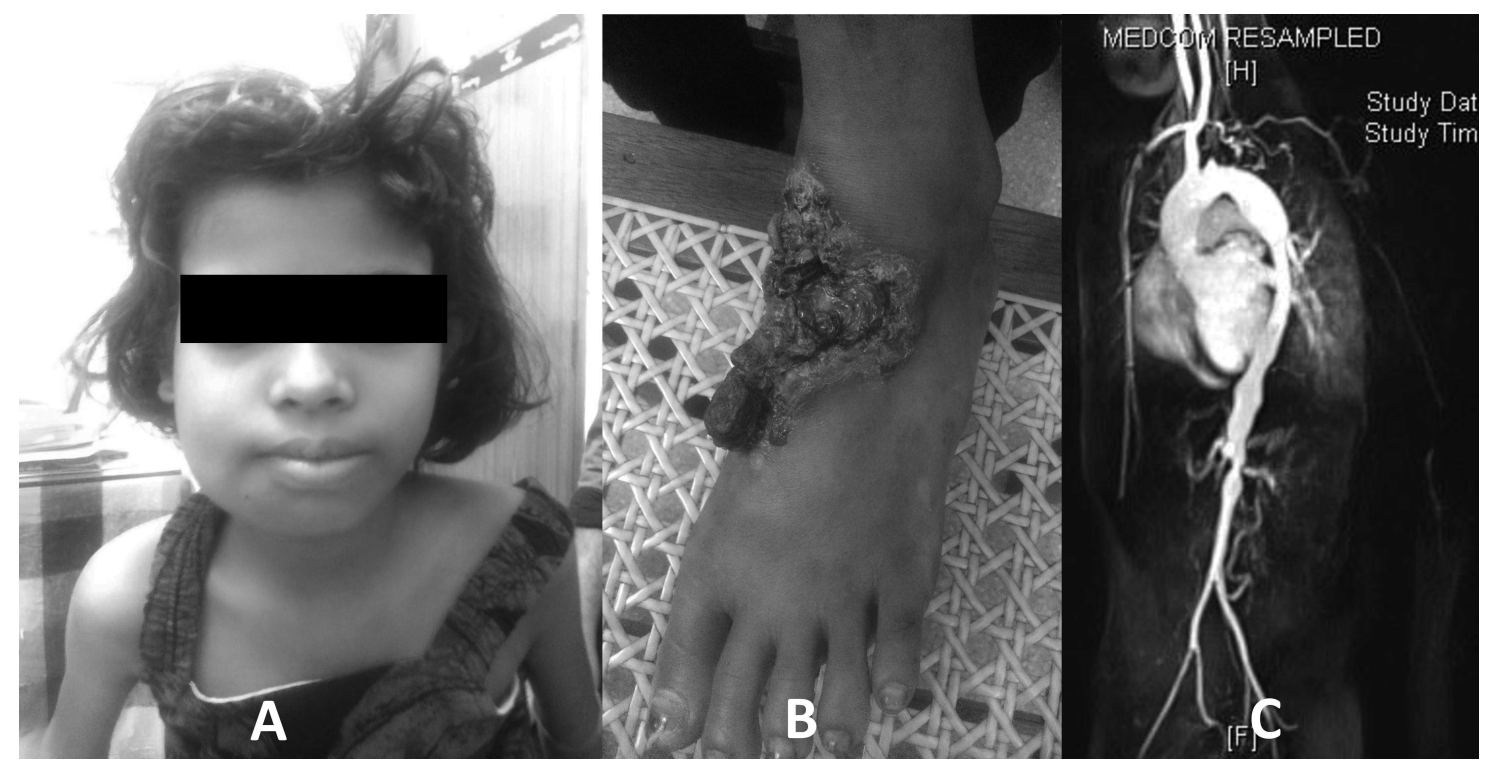

Figure 1. A. Swelling over the right side of mandible. B. Cauliflower-like skin lesion was seen over dorsum of left foot. C. Descending thoracic aorta showing long segment of mild narrowing at lower thoracic aorta with some areas of mild dilation just above the level of the diaphragm. 


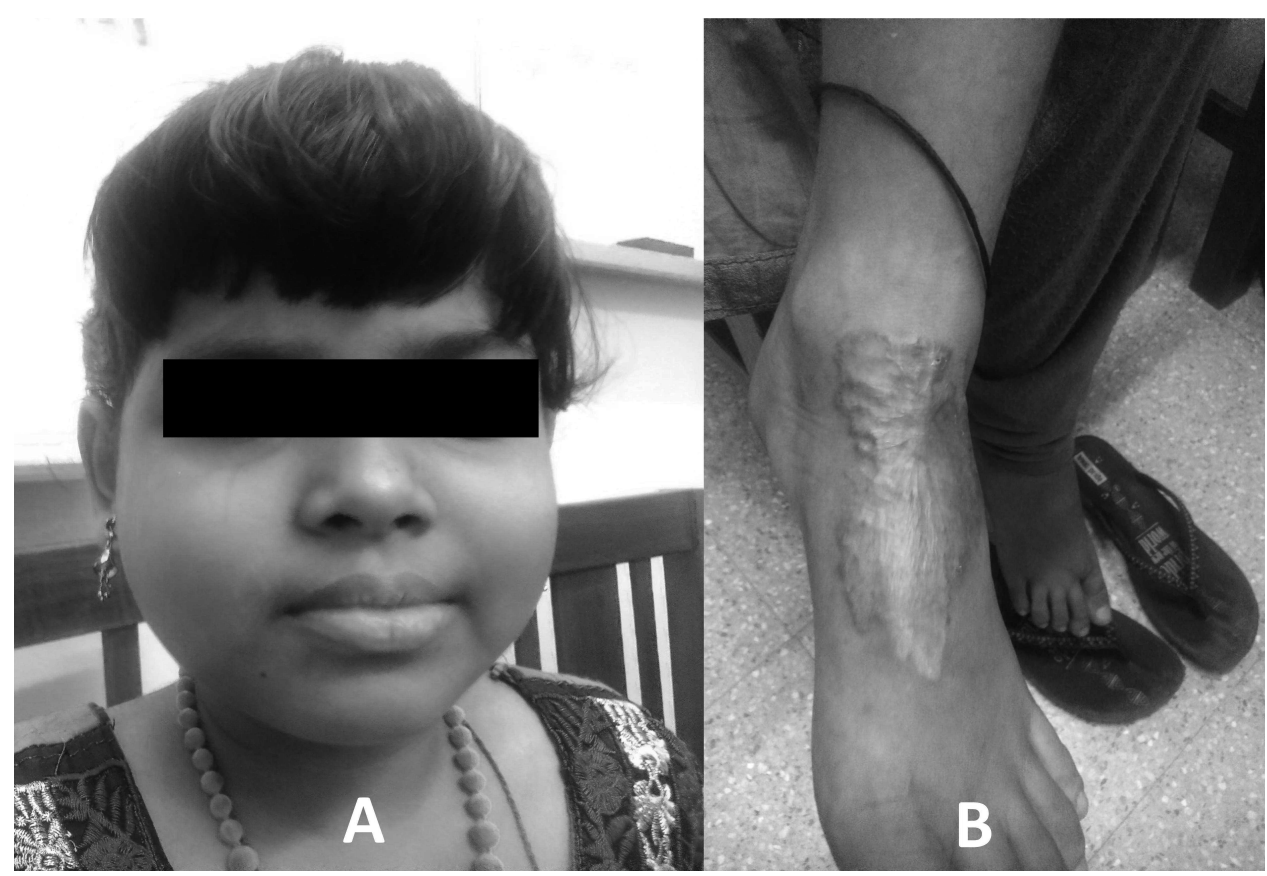

Figure 2. A. Resolution of lesion over the right side of mandible. B. Healing of pyoderma gangrenosum over dorsum of left foot.

GEORGE VETTIYIL, DCH, Christian Medical College and Hospital Vellore, Pediatric Unit II; ANU PUNNEN, MD, Christian Medical College and Hospital Vellore, Child Health II, Assistant Professor, Department of Pediatrics; SATHISH KUMAR, MD, DCH, Christian Medical College, Paediatrics, Department of Pediatrics, Christian Medical College, Vellore, India. Address correspondence to Dr. S. Kumar, Christian Medical College, Department of Pediatrics, Christian Medical College, Vellore, Tamil Nadu 632004, India. E-mail: sathishkumar@cmcvellore.ac.in

\section{REFERENCES}

1. Roderick MR, Ramanan AV. Chronic recurrent multifocal osteomyelitis. Adv Exp Med Biol 2013;764:99-107.

2. Ferguson PJ, Sandu M. Current understanding of the pathogenesis and management of chronic recurrent multifocal osteomyelitis. Curr Rheumatol Rep 2012;14:130-41.
3. Ujiie H, Sawamura D, Yokota K, Nishie W, Shichinohe R, Shimizu H. Pyoderma gangrenosum associated with Takayasu's arteritis. Clin Exp Dermatol 2004;29:357-9.

4. Dagan O, Barak Y, Metzker A. Pyoderma gangrenosum and sterile multifocal osteomyelitis preceding the appearance of Takayasu arteritis. Pediatr Dermatol 1995;12:39-42.

5. Ghosn S, Malek J, Shbaklo Z, Matta M, Uthman I. Takayasu disease presenting as malignant pyoderma gangrenosum in a child with relapsing polychondritis. J Am Acad Dermatol 2008;59 Suppl 5:S84-7.

J Rheumatol 2017;44:1; doi:10.3899/jrheum.160491 